\title{
A Fractional Order Model for Viral Infection with Cure of Infected Cells and Humoral Immunity
}

\author{
Adnane Boukhouima, ${ }^{1}$ Khalid Hattaf $\mathbb{D}^{1},{ }^{1,2}$ and Noura Yousfi $(\mathbb{D})^{1}$ \\ ${ }^{1}$ Laboratory of Analysis, Modeling and Simulation (LAMS), Faculty of Sciences Ben M'sik, Hassan II University, \\ P.O Box 7955 Sidi Othman, Casablanca, Morocco \\ ${ }^{2}$ Centre Régional des Métiers de l'Education et de la Formation (CRMEF), 20340 Derb Ghalef, Casablanca, Morocco
}

Correspondence should be addressed to Khalid Hattaf; k.hattaf@yahoo.fr

Received 9 September 2018; Accepted 7 November 2018; Published 2 December 2018

Guest Editor: Nurcan B. Savaşaneril

Copyright (C) 2018 Adnane Boukhouima et al. This is an open access article distributed under the Creative Commons Attribution License, which permits unrestricted use, distribution, and reproduction in any medium, provided the original work is properly cited.

In this paper, we study the dynamics of a viral infection model formulated by five fractional differential equations (FDEs) to describe the interactions between host cells, virus, and humoral immunity presented by antibodies. The infection transmission process is modeled by Hattaf-Yousfi functional response which covers several forms of incidence rate existing in the literature. We first show that the model is mathematically and biologically well-posed. By constructing suitable Lyapunov functionals, the global stability of equilibria is established and characterized by two threshold parameters. Finally, some numerical simulations are presented to illustrate our theoretical analysis.

\section{Introduction}

The immune response plays an important role to control the dynamics of viral infections such as human immunodeficiency virus (HIV), hepatitis B virus (HBV), hepatitis C virus (HCV), and human T-cell leukemia virus (HTLV). Therefore, many mathematical models have been developed to incorporate the role of immune response in viral infections. Some of these models considered the cellular immune response mediated by cytotoxic T lymphocytes (CTL) cells that attack and kill the infected cells [1-5] and the others considered the humoral immune response based on the antibodies which are produced by the B-cells and are programmed to neutralize the viruses [6-11]. However, all these models have been formulated by using ordinary differential equations (ODEs) in which the memory effect is neglected while the immune response involves memory $[12,13]$.

Fractional derivative is a generalization of integer derivative and it is a suitable tool to model real phenomena with memory which exists in most biological systems [14-16]. The fractional derivative is a nonlocal operator in contrast to integer derivative. This means that if we want to compute the fractional derivative at some point $t=t_{1}$, it is necessary to take into account the entire history from the starting point $t=t_{0}$ up to the point $t=t_{1}$. For these reasons, modeling some real process by using fractional derivative has drawn attention of several authors in various fields [17-22]. In biology, it has been shown that the fractional derivative is useful to analyse the rheological proprieties of cells [23]. Furthermore, it has been deduced that the membranes of cells of biological organism have fractional order electrical conductance [24]. Recently, much works have been done on modeling the dynamics of viral infections with FDEs [25-31]. These works ignored the impact of the immune response and the majority of them deal only with the local stability.

In some viral infections, the humoral immune response is more effective than cellular immune response [32]. For this reason, we improve the above ODE and FDE models by proposing a new fractional order model that describes the interactions between susceptible host cells, viral particles, and the humoral immune response mediated by the antibodies; that is,

$$
\begin{aligned}
D^{\alpha} x(t) & =\lambda-d x-f(x, v) v+\rho l, \\
D^{\alpha} l(t) & =f(x, v) v-(m+\rho+\gamma) l,
\end{aligned}
$$




$$
\begin{aligned}
D^{\alpha} y(t) & =\gamma l-a y, \\
D^{\alpha} v(t) & =k y-\mu v-q v w, \\
D^{\alpha} w(t) & =g v w-h w,
\end{aligned}
$$

where $x(t), l(t), y(t), v(t)$, and $w(t)$ are the concentrations of susceptible host cells, latently infected cells (infected cells which are not yet able to produce virions), productive infected cells, free virus particles, and antibodies at time $t$, respectively. Susceptible host cells are assumed to be produced at a constant rate $\lambda$, die at the rate $d x$, and become infected by virus at the rate $f(x, v) v$. Latently infected cells die at the rate $m l$ and return to the uninfected state by loss of all covalently closed circular DNA (cccDNA) from their nucleus at the rate $\rho l$. Productive infected cells are produced from latently infected cells at the rate $\gamma l$ and die at the rate $a y$. Free virus particles are produced from productive infected cells at the rate $k y$, cleared at the rate $\mu v$, and are neutralized by antibodies at the rate $q v w$. Antibodies are activated against virus at the rate $g v w$ and die at the rate $h w$.

In system (1), $D^{\alpha}$ represents the Caputo fractional derivative of order $\alpha$ defined for an arbitrary function $\varphi$ by

$$
D^{\alpha} \varphi(t)=\frac{1}{\Gamma(1-\alpha)} \int_{0}^{t} \frac{\varphi^{\prime}(u)}{(t-u)^{\alpha}} d u
$$

with $0<\alpha \leq 1$ [33]. Further, the infection transmission process in (1) is modeled by Hattaf-Yousfi functional response [34] which was recently used in $[35,36]$ and has the form $f(x, v)=\beta x /\left(\alpha_{0}+\alpha_{1} x+\alpha_{2} v+\alpha_{3} x v\right)$, where $\alpha_{0}, \alpha_{1}, \alpha_{2}, \alpha_{3} \geq$ 0 are the saturation factors measuring the psychological or inhibitory effect and $\beta>0$ is the infection rate. In addition, this functional response generalizes many common types existing in the literature such as the specific functional response proposed by Hattaf et al. in [37] and used in [2, 31] when $\alpha_{0}=1$; the Crowley-Martin functional response introduced in [38] and used in [39] when $\alpha_{0}=1$ and $\alpha_{3}=$ $\alpha_{1} \alpha_{2}$; and the Beddington-DeAngelis functional response proposed in $[40,41]$ and used in $[3,4,10]$ when $\alpha_{0}=1$ and $\alpha_{3}=0$. Also, the Hattaf-Yousfi functional response is reduced to the saturated incidence rate used in [9] when $\alpha_{0}=1$ and $\alpha_{1}=\alpha_{3}=0$ and the standard incidence function used in [27] when $\alpha_{0}=\alpha_{3}=0$ and $\alpha_{1}=\alpha_{2}=1$, and it was simplified to the bilinear incidence rate used in $[5,6]$ when $\alpha_{0}=1$ and $\alpha_{1}=\alpha_{2}=\alpha_{3}=0$.

On the other hand, system (1) becomes a model with ODEs when $\alpha=1$, which improves and generalizes the ODE model with bilinear incidence rate [42], the ODE model with saturated incidence rate [43], and the ODE model with specific functional response [44].

The rest of the paper is organized as follows. The next section deals with some basic proprieties of the solutions and the existence of equilibria. The global stability of equilibria is established in Section 3. To verify our theoretical results, we provide some numerical simulations in Section 4 , and we conclude in Section 5.

\section{Basic Properties and Equilibria}

In this section, we will show that our model is well-posed and we discuss the existence of equilibria.

Since system (1) describes the evolution of cells, then we need to prove that the cell numbers should remain nonnegative and bounded. For biological considerations, we assume that the initial conditions of (1) satisfy

$$
\begin{aligned}
& x(0) \geq 0, \\
& l(0) \geq 0, \\
& y(0) \geq 0, \\
& v(0) \geq 0, \\
& w(0) \geq 0 .
\end{aligned}
$$

Then we have the following result.

Theorem 1. Assume that the initial conditions satisfy (3). Then there exists a unique solution of system (1) defined on $[0,+\infty)$. Moreover, this solution remains nonnegative and bounded for all $t \geq 0$.

Proof. First, system (1) can be written as follows:

$$
D^{\alpha} X(t)=F(X),
$$

where

$$
X(t)=\left(\begin{array}{c}
x(t) \\
l(t) \\
y(t) \\
v(t) \\
w(t)
\end{array}\right)
$$

$$
\text { and } F(X)=\left(\begin{array}{c}
\lambda-d x-f(x, v) v+\rho l \\
f(x, v) v-(m+\rho+\gamma) l \\
\gamma l-a y \\
k y-\mu v-q v w \\
g v w-h w
\end{array}\right) \text {. }
$$

It is important to note that when $\alpha=1$, (4) becomes a system with ODEs. In this case, we refer the reader to [45] for the existence of solutions and to the works [46-50] for the stability of equilibria. In the case of FDEs, we will use Lemma 
2.4 in [31] to prove the existence and uniqueness of solutions. Hence, we put

$$
\begin{aligned}
\zeta & =\left(\begin{array}{c}
\lambda \\
0 \\
0 \\
0 \\
0
\end{array}\right), \\
A & \left.=\left(\begin{array}{ccccc}
-d & \rho & 0 & 0 & 0 \\
0 & -(m+\rho+\gamma) & 0 & 0 & 0 \\
0 & & \gamma \\
0 & & 0 \\
0 & & 0 & 0 & 0 \\
\text { and } C & = \\
0 & 0 & 0 & 0 & 0 \\
0 & 0 & 0 & 0 & 0 \\
0 & 0 & 0 & 0 & 0 \\
0 & 0 & 0 & 0 & -q \\
0 & 0 & 0 & 0 & g
\end{array}\right) \cdot \begin{array}{ccc}
0 \\
0
\end{array}\right)
\end{aligned}
$$

We discuss four cases:

(i) If $\alpha_{0} \neq 0, F(X)$ can be formulated as follows:

$$
\begin{aligned}
F(X)= & \zeta+A X+\frac{\alpha_{0}}{\alpha_{0}+\alpha_{1} x+\alpha_{2} v+\alpha_{3} x v} v B_{0} X \\
& +v C X,
\end{aligned}
$$

where

$$
B_{0}=\left(\begin{array}{ccccc}
-\frac{\beta}{\alpha_{0}} & 0 & 0 & 0 & 0 \\
\frac{\beta}{\alpha_{0}} & 0 & 0 & 0 & 0 \\
0 & 0 & 0 & 0 & 0 \\
0 & 0 & 0 & 0 & 0 \\
0 & 0 & 0 & 0 & 0
\end{array}\right)
$$

Hence,

$$
\|F(X)\| \leq\|\zeta\|+\left(\|A\|+\|v\|\left(\left\|B_{0}\right\|+\|C\|\right)\right)\|X\|
$$

(ii) If $\alpha_{1} \neq 0$, we can write $F(X)$ in the form

$$
\begin{aligned}
F(X)= & \zeta+A X+\frac{\alpha_{1} x}{\alpha_{0}+\alpha_{1} x+\alpha_{2} v+\alpha_{3} x v} B_{1} X \\
& +v C X
\end{aligned}
$$

where

$$
B_{1}=\left(\begin{array}{ccccc}
0 & 0 & 0 & -\frac{\beta}{\alpha_{1}} & 0 \\
0 & 0 & 0 & \frac{\beta}{\alpha_{1}} & 0 \\
0 & 0 & 0 & 0 & 0 \\
0 & 0 & 0 & 0 & 0 \\
0 & 0 & 0 & 0 & 0
\end{array}\right) .
$$

Moreover, we get

$$
\|F(X)\| \leq\|\zeta\|+\left(\|A\|+\left\|B_{1}\right\|+\|v\|\|C\|\right)\|X\| .
$$

(iii) If $\alpha_{2} \neq 0$, we have

$$
\begin{aligned}
F(X)= & \zeta+A X+\frac{\alpha_{2} v}{\alpha_{0}+\alpha_{1} x+\alpha_{2} v+\alpha_{3} x v} B_{2} X \\
& +v C X,
\end{aligned}
$$

where

$$
B_{2}=\left(\begin{array}{ccccc}
-\frac{\beta}{\alpha_{2}} & 0 & 0 & 0 & 0 \\
\frac{\beta}{\alpha_{2}} & 0 & 0 & 0 & 0 \\
0 & 0 & 0 & 0 & 0 \\
0 & 0 & 0 & 0 & 0 \\
0 & 0 & 0 & 0 & 0
\end{array}\right) .
$$

Further, we obtain

$$
\|F(X)\| \leq\|\zeta\|+\left(\|A\|+\left\|B_{2}\right\|+\|v\|\|C\|\right)\|X\| .
$$

(iv) If $\alpha_{3} \neq 0$, we have

$$
\begin{gathered}
F(X)=\zeta+A X+\frac{\alpha_{3} x v}{\alpha_{0}+\alpha_{1} x+\alpha_{2} v+\alpha_{3} x v} B_{3}+v C X, \\
\text { where }
\end{gathered}
$$

$$
B_{3}=\left(\begin{array}{c}
-\frac{\beta}{\alpha_{3}} \\
\frac{\beta}{\alpha_{3}} \\
0 \\
0 \\
0
\end{array}\right)
$$

Then

$$
\|F(X)\| \leq\left(\|\zeta\|+\left\|B_{3}\right\|\right)+(\|A\|+\|v\|\|C\|)\|X\| .
$$


Hence, the conditions of Lemma 2.4 in [31] are verified. Then system (1) has a unique solution on $[0,+\infty)$. Now, we show the nonnegativity of solutions. By (1), we have

$$
\begin{aligned}
& \left.D^{\alpha} x(t)\right|_{x=0}=\lambda+\rho l \geq 0, \\
& \left.D^{\alpha} l(t)\right|_{l=0}=f(x, v) v \geq 0, \\
& \left.D^{\alpha} y(t)\right|_{y=0}=\gamma l \geq 0, \\
& \left.D^{\alpha} v(t)\right|_{v=0}=k y \geq 0, \\
& \left.D^{\alpha} w(t)\right|_{w=0}=0 \geq 0 .
\end{aligned}
$$

As in [31, Theorem 2.7], we deduce that the solution of (1) is nonnegative.

Finally, we prove the boundedness of solutions. We define the function

$$
T(t)=x(t)+l(t)+y(t)+\frac{a}{2 k} v(t)+\frac{a q}{2 k g} w(t) .
$$

Then, we have

$$
\begin{aligned}
D^{\alpha} T(t)= & D^{\alpha} x(t)+D^{\alpha} l(t)+D^{\alpha} y(t)+\frac{a}{2 k} D^{\alpha} v(t) \\
& +\frac{a q}{2 k g} D^{\alpha} w(t) \\
= & \lambda-d x(t)-m l(t)-\frac{a}{2} y(t)-\frac{a \mu}{2 k} v(t) \\
& -\frac{a q h}{2 k g} w(t) \leq \lambda-\delta T(t),
\end{aligned}
$$

where $\delta=\min \{d, m, a / 2, \mu, h\}$. Thus, we obtain

$$
T(t) \leq T(0) E_{\alpha}\left(-\delta t^{\alpha}\right)+\frac{\lambda}{\delta}\left[1-E_{\alpha}\left(-\delta t^{\alpha}\right)\right] .
$$

Since $0 \leq E_{\alpha}\left(-\delta t^{\alpha}\right) \leq 1$, we get

$$
T(t) \leq T(0)+\frac{\lambda}{\delta}
$$

This completes the proof.

Now, we discuss the existence of equilibria. It is clear that system (1) has always an infection-free equilibrium $E_{0}(\lambda / d, 0,0,0,0)$. Then the basic reproduction number of (1) is as follows:

$$
R_{0}=\frac{k \beta \lambda \gamma}{a \mu(m+\rho+\gamma)\left(d \alpha_{0}+\lambda \alpha_{1}\right)} .
$$

To find the other equilibria, we solve the following system:

$$
\begin{aligned}
\lambda-d x-f(x, v) v+\rho l & =0, \\
f(x, v) v-(m+\rho+\gamma) l & =0, \\
\gamma l-a y & =0, \\
k y-\mu v-q v w & =0, \\
g v w-h w & =0 .
\end{aligned}
$$

From (29), we get $w=0$ or $v=h / g$. Then we discuss two cases.

If $w=0$, by (25)-(28), we have $l=(\lambda-d x) /(m+\gamma)$, $y=\gamma(\lambda-d x) / a(m+\gamma), v=k \gamma(\lambda-d x) / a \mu(m+\gamma)$, and

$$
f\left(x, \frac{k \gamma(\lambda-d x)}{a \mu(m+\gamma)}\right)=\frac{a \mu(m+\rho+\gamma)}{k \gamma} .
$$

Since $l \geq 0, y \geq 0$, and $v \geq 0$, then $x \leq \lambda / d$. Consequently, there is no equilibrium when $x>\lambda / d$.

We define the function $h_{1}$ on $[0, \lambda / d]$ by

$$
h_{1}(x)=f\left(x, \frac{k \gamma(\lambda-d x)}{a \mu(m+\gamma)}\right)-\frac{a \mu(m+\rho+\gamma)}{k \gamma} .
$$

We have $h_{1}(0)=-a \mu(m+\rho+\gamma) / k \gamma<0, h_{1}^{\prime}(x)=\partial f / \partial x-$ $(k \gamma d / a \mu(m+\gamma))(\partial f / \partial v)>0$, and $h_{1}(\lambda / d)=(a \mu(m+\rho+$ $\gamma) / k \gamma)\left(R_{0}-1\right)$.

Hence if $R_{0}>1,(30)$ has a unique root $x_{1} \in(0, \lambda / d)$. As a result, when $R_{0}>1$ there exists an equilibrium $E_{1}\left(x_{1}, l_{1}, y_{1}, v_{1}, 0\right)$ satisfying $x_{1} \in(0, \lambda / d), l_{1}=\left(\lambda-d x_{1}\right) /(m+$ $\gamma), y_{1}=\gamma\left(\lambda-d x_{1}\right) / a(m+\gamma)$, and $v_{1}=k \gamma\left(\lambda-d x_{1}\right) / a \mu(m+\gamma)$.

If $w \neq 0$, then $v=h / g$. By (25)-(27), we obtain $l=(\lambda-$ $d x) /(m+\gamma), y=\gamma(\lambda-d x) / a(m+\gamma), w=k \gamma g(\lambda-d x) / \operatorname{aqh}(m+$ $\gamma)-\mu / q$, and

$$
f\left(x, \frac{h}{g}\right)=\frac{g(m+\rho+\gamma)}{h(m+\gamma)}(\lambda-d x) .
$$

Since $l \geq 0, y \geq 0$, and $w \geq 0$, we have $x \leq \lambda / d-\operatorname{ah} \mu(m+$ $\gamma) / d k g \gamma$. Hence, there is no equilibrium if $x>\lambda / d-a h \mu(m+$ $\gamma) / d k g \gamma$. by

We define the function $h_{2}$ on $[0, \lambda / d-a h \mu(m+\gamma) / d k g \gamma]$

$$
h_{2}(x)=f\left(x, \frac{h}{g}\right)-\frac{g(m+\rho+\gamma)}{h(m+\gamma)}(\lambda-d x) .
$$

We have $h_{2}(0)=-g \lambda(m+\rho+\gamma) / h(m+\gamma)<0, h_{2}^{\prime}(x)=$ $\partial f / \partial x+g d(m+\rho+\gamma) / h(m+\gamma)>0$, and $h_{2}(\lambda / d-a h \mu(m+$ $\gamma) / d k g \gamma)=h_{1}(\lambda / d-a h \mu(m+\gamma) / d k g \gamma)$.

Let us introduce the reproduction number for humoral immunity as follows:

$$
R_{1}=\frac{g v_{1}}{h}
$$

which $1 / h$ denotes the average life expectancy of antibodies and $v_{1}$ is the number of free viruses at $E_{1}$. For the biological significance, $R_{1}$ represents the average number of the antibodies activated by virus.

If $R_{1}<1$, we have $x_{1}>\lambda / d-a h \mu(m+\gamma) / d k g \gamma$ and

$$
h_{2}\left(\frac{\lambda}{d}-\frac{a h \mu(m+\gamma)}{d k g \gamma}\right)<h_{1}\left(x_{1}\right)=0 .
$$

Therefore, there is no equilibrium when $R_{1}<1$.

If $R_{1}>1$, then $x_{1}<\lambda / d-a h \mu(m+\gamma) / d k g \gamma$ and

$$
h_{2}\left(\frac{\lambda}{d}-\frac{a h \mu(m+\gamma)}{d k g \gamma}\right)>h_{1}\left(x_{1}\right)=0 .
$$


In this case, (32) has one root $x_{2} \in(0, \lambda / d-a h \mu(m+\gamma) / d k g \gamma)$. Consequently, when $R_{1}>1$, there exists an equilibrium $E_{2}\left(x_{2}, l_{2}, y_{2}, v_{2}, w_{2}\right)$ satisfying $x_{2} \in(0, \lambda / d-\operatorname{ah} \mu(m+$ $\gamma) / d k g \gamma), l_{2}=\left(\lambda-d x_{2}\right) /(m+\gamma), y_{2}=\gamma\left(\lambda-d x_{2}\right) / a(m+\gamma)$, $v_{2}=h / g$, and $w_{2}=k \gamma g\left(\lambda-d x_{2}\right) / \operatorname{aqh}(m+\gamma)-\mu / q$. When $R_{1}=1, E_{1}=E_{2}$.

We summarize the above discussions in the following theorem.

\section{Theorem 2.}

(i) If $R_{0} \leq 1$, then system (1) has one infection-free equilibrium of the form $E_{0}\left(x_{0}, 0,0,0,0\right)$, where $x_{0}=$ $\lambda / d$.

(ii) If $R_{0}>1$, then system (1) has an infection equilibrium without humoral immunity of the form $E_{1}\left(x_{1}, l_{1}, y_{1}, v_{1}, 0\right)$, where $x_{1} \in(0, \lambda / d), l_{1}=(\lambda-$ $\left.d x_{1}\right) /(m+\gamma), y_{1}=\gamma\left(\lambda-d x_{1}\right) / a(m+\gamma)$, and $v_{1}=$ $k \gamma\left(\lambda-d x_{1}\right) / a \mu(m+\gamma)$.

(iii) If $R_{1}>1$, then system (1) has an infection equilibrium with humoral immunity of the form $E_{2}\left(x_{2}, l_{2}, y_{2}, v_{2}, w_{2}\right)$, where $x_{2} \in(0, \lambda / d-\operatorname{ah\mu }(m+$ $\gamma) / d k g \gamma), l_{2}=\left(\lambda-d x_{2}\right) /(m+\gamma), y_{2}=\gamma\left(\lambda-d x_{2}\right) / a(m+$ $\gamma), v_{2}=h / g$, and $w_{2}=k \gamma g\left(\lambda-d x_{1}\right) / a q h(m+\gamma)-\mu / q$.

\section{Global Stability of Equilibria}

In this section, we focus on the global stability of equilibria.

Theorem 3. If $R_{0} \leq 1$, then the infection-free equilibrium $E_{0}$ is globally asymptotically stable and it becomes unstable if $R_{0}>1$.

Proof. The proof of the first part of this theorem is based on the construction of a suitable Lyapunov functional that satisfies the conditions given in [51, Lemma 4.6]. Hence, we define a Lyapunov functional as follows:

$$
\begin{aligned}
& L_{0}(t) \\
& =\frac{\alpha_{0}}{\alpha_{0}+\alpha_{1} x_{0}} x_{0} \Phi\left(\frac{x}{x_{0}}\right) \\
& +\frac{\rho \alpha_{0}}{2(d+m+\gamma)\left(\alpha_{0}+\alpha_{1} x_{0}\right) x_{0}}\left(x-x_{0}+l\right)^{2} \\
& +l+\frac{m+\rho+\gamma}{\gamma} y+\frac{a(m+\rho+\gamma)}{k \gamma} v \\
& +\frac{a q(m+\rho+\gamma)}{k g \gamma} w
\end{aligned}
$$

where $\Phi(x)=x-1-\ln (x)$ for $x>0$. It is not hard to show that the functional $L_{0}$ is nonnegative. In fact, the function $\Phi$ has a global minimum at $x=1$. Consequently, $\Phi(x) \geq 0$ for all $x>0$.
Calculating the fractional derivative of $L_{0}(t)$ along solutions of system (1) and using the results in [52], we get

$$
\begin{aligned}
& D^{\alpha} L_{0}(t) \leq \frac{\alpha_{0}}{\alpha_{0}+\alpha_{1} x_{0}}\left(1-\frac{x_{0}}{x}\right) D^{\alpha} x \\
& +\frac{\rho \alpha_{0}}{(d+m+\gamma)\left(\alpha_{0}+\alpha_{1} x_{0}\right) x_{0}}\left(x-x_{0}+l\right) \\
& \cdot\left(D^{\alpha} x+D^{\alpha} l\right)+D^{\alpha} l+\frac{m+\rho+\gamma}{\gamma} D^{\alpha} y \\
& +\frac{a(m+\rho+\gamma)}{k \gamma} D^{\alpha} v+\frac{a q(m+\rho+\gamma)}{k g \gamma} D^{\alpha} w .
\end{aligned}
$$

Using $\lambda=d x_{0}$, we obtain

$$
\begin{aligned}
& D^{\alpha} L_{0}(t) \leq-\frac{d \alpha_{0}\left(x-x_{0}\right)^{2}}{\left(\alpha_{0}+\alpha_{1} x_{0}\right) x}-\frac{\alpha_{0}}{\alpha_{0}+\alpha_{1} x_{0}}\left(1-\frac{x_{0}}{x}\right) \\
& \cdot f(x, v) v+\frac{\rho \alpha_{0}}{\alpha_{0}+\alpha_{1} x_{0}}\left(1-\frac{x_{0}}{x}\right) l \\
& \cdot \frac{\rho \alpha_{0}\left(x-x_{0}+l\right)}{(d+m+\gamma)\left(\alpha_{0}+\alpha_{1} x_{0}\right) x_{0}}\left(d\left(x_{0}-x\right)\right. \\
& -(m+\gamma) l)+f(x, v) v-\frac{a \mu(m+\rho+\gamma)}{k \gamma} v \\
& -\frac{a q h(m+\rho+\gamma)}{k g \gamma} w \\
& \leq-\left(\frac{1}{x}+\frac{\rho}{(d+m+\gamma) x_{0}}\right) \frac{d \alpha_{0}\left(x-x_{0}\right)^{2}}{\left(\alpha_{0}+\alpha_{1} x_{0}\right)} \\
& +\frac{\rho \alpha_{0}(m+\gamma) l^{2}}{(d+m+\gamma)\left(\alpha_{0}+\alpha_{1} x_{0}\right) x_{0}}-\frac{\rho \alpha_{0}\left(x-x_{0}\right)^{2} l}{\left(\alpha_{0}+\alpha_{1} x_{0}\right) x x_{0}} \\
& +\frac{a \mu(m+\rho+\gamma)}{k \gamma}\left(R_{0}-1\right) v-\frac{a q h(m+\rho+\gamma)}{k g \gamma} w .
\end{aligned}
$$

Hence if $R_{0} \leq 1$, then $D^{\alpha} L_{0}(t) \leq 0$. In addition, the equality holds if and only if $x=x_{0}, l=0, y=0, w=0$, and $\left(R_{0}-1\right) v=$ 0 . If $R_{0}<1$, then $v=0$. If $R_{0}=1$, from (1), we get $f\left(x_{0}, v\right) v=$ 0 which implies that $v=0$. Consequently, the largest invariant set of $\left\{(x, l, y, v, w) \in \mathbb{R}_{+}^{5}: D^{\alpha} L_{0}(t)=0\right\}$ is the singleton $\left\{E_{0}\right\}$. Therefore, by the LaSalle's invariance principle [51], $E_{0}$ is globally asymptotically stable.

The proof of the instability of $E_{0}$ is based on the computation of the Jacobean matrix of system (1) and the results presented in [53-55]. The Jacobean matrix of (1) at any equilibrium $E(x, l, y, v, w)$ is given by

$$
\left(\begin{array}{ccccc}
-d-\frac{\partial f}{\partial x} v & \rho & 0 & -\frac{\partial f}{\partial v} v-f(x, v) & 0 \\
\frac{\partial f}{\partial x} v & -(m+\rho+\gamma) & 0 & \frac{\partial f}{\partial v} v+f(x, v) & 0 \\
0 & \gamma & -a & 0 & 0 \\
0 & 0 & k & -\mu-q w & -q v \\
0 & 0 & 0 & g w & g v-h
\end{array}\right)
$$


We recall that $E$ is locally asymptotically stable if the all eigenvalues $\xi_{i}$ of (40) satisfy the following condition [53-55]:

$$
\left|\arg \left(\xi_{i}\right)\right|>\frac{\alpha \pi}{2}
$$

From (40), the characteristic equation at $E_{0}$ is given as follows:

$$
(d+\xi)(h+\xi) g_{0}(\xi)=0
$$

where

$$
\begin{aligned}
g_{0}(\xi)= & ((m+\rho+\gamma)+\xi)(a+\xi)(\mu+\xi) \\
& -\frac{k \gamma \beta \lambda}{d \alpha_{0}+\alpha_{1} \lambda} .
\end{aligned}
$$

Obviously, (42) has the roots $\xi_{1}=-d$ and $\xi_{2}=-h$. If $R_{0}>1$, we have $g_{0}(0)=a \mu(m+\rho+\gamma)\left(1-R_{0}\right)<0$ and $\lim _{\xi \rightarrow+\infty} g_{0}(\xi)=+\infty$. Then, there exists $\xi^{*}>0$ satisfying $g_{0}\left(\xi^{*}\right)=0$. In addition, we have $\left|\arg \left(\xi^{*}\right)\right|=0<\alpha \pi / 2$. Consequently, when $R_{0}>1, E_{0}$ is unstable.

\section{Theorem 4.}

(i) The infection equilibrium without humoral immunity $E_{1}$ is globally asymptotically stable if $R_{0}>1, R_{1} \leq 1$, and

$$
\begin{aligned}
R_{0} & \leq 1 \\
& +\frac{(m+\rho+\gamma)\left[\alpha_{0} a d \mu(m+\rho)+d k \lambda \gamma \alpha_{2}\right]+k \rho \gamma \alpha_{3} \lambda^{2}}{a \rho \mu(m+\rho+\gamma)\left(\alpha_{0} d+\lambda \alpha_{1}\right)} .
\end{aligned}
$$

(ii) When $R_{1}>1, E_{1}$ is unstable.
Proof. Define a Lyapunov functional as follows:

$$
\begin{aligned}
& L_{1}(t)=\frac{\alpha_{0}+\alpha_{2} v_{1}}{\alpha_{0}+\alpha_{1} x_{1}+\alpha_{2} v_{1}+\alpha_{3} x_{1} v_{1}} x_{1} \Phi\left(\frac{x}{x_{1}}\right) \\
& +l_{1} \Phi\left(\frac{l}{l_{1}}\right) \\
& +\frac{\rho\left(\alpha_{0}+\alpha_{2} v_{1}\right)}{2(d+m+\gamma)\left(\alpha_{0}+\alpha_{1} x_{1}+\alpha_{2} v_{1}+\alpha_{3} x_{1} v_{1}\right) x_{1}}(x) \\
& \left.\quad-x_{1}+l-l_{1}\right)^{2}+\frac{m+\rho+\gamma}{\gamma} y_{1} \Phi\left(\frac{y}{y_{1}}\right) \\
& +\frac{a(m+\rho+\gamma)}{k \gamma} v_{1} \Phi\left(\frac{v}{v_{1}}\right)+\frac{a q(m+\rho+\gamma)}{k g \gamma} w .
\end{aligned}
$$

Calculating the fractional derivative of $L_{1}(t)$, we get

$$
\begin{aligned}
& D^{\alpha} L_{1}(t)=\frac{\alpha_{0}+\alpha_{2} v_{1}}{\alpha_{0}+\alpha_{1} x_{1}+\alpha_{2} v_{1}+\alpha_{3} x_{1} v_{1}}\left(1-\frac{x_{1}}{x}\right) D^{\alpha} x \\
& +\left(1-\frac{l_{1}}{l}\right) D^{\alpha} l \\
& +\frac{\rho\left(\alpha_{0}+\alpha_{2} v_{1}\right)\left(x-x_{1}+l-l_{1}\right)}{(d+m+\gamma)\left(\alpha_{0}+\alpha_{1} x_{1}+\alpha_{2} v_{1}+\alpha_{3} x_{1} v_{1}\right) x_{1}}\left(D^{\alpha} x\right. \\
& \left.+D^{\alpha} l\right)+\frac{m+\rho+\gamma}{\gamma}\left(1-\frac{y_{1}}{y}\right) D^{\alpha} y \\
& +\frac{a(m+\rho+\gamma)}{k \gamma}\left(1-\frac{v_{1}}{v}\right) D^{\alpha} v+\frac{a q(m+\rho+\gamma)}{k g \gamma} w .
\end{aligned}
$$

Using $\lambda=d x_{1}+(m+\gamma) l_{1}, f\left(x_{1}, v_{1}\right) v_{1}=(m+\rho+\gamma) l_{1}, \gamma l_{1}=a y_{1}$, $k y_{1}=\mu v_{1}$, and $1-f\left(x_{i}, v_{i}\right) / f\left(x, v_{i}\right)=\left(\left(\alpha_{0}+\alpha_{2} v_{i}\right) /\left(\alpha_{0}+\alpha_{1} x_{i}+\right.\right.$ $\left.\left.\alpha_{2} v_{i}+\alpha_{3} x_{i} v_{i}\right)\right)\left(1-x_{i} / x\right) \forall i \in\{1,2\}$, we obtain

$$
\begin{aligned}
D^{\alpha} L_{1}(t) \leq & d\left(1-\frac{f\left(x_{1}, v_{1}\right)}{f\left(x, v_{1}\right)}\right)\left(x_{1}-x\right)+(m+\rho+\gamma) l_{1}\left(1-\frac{f\left(x_{1}, v_{1}\right)}{f\left(x, v_{1}\right)}+\frac{v}{v_{1}} \frac{f(x, v)}{f\left(x, v_{1}\right)}\right)+(m+\rho+\gamma) \\
& \cdot l_{1}\left(1-\frac{l_{1} f(x, v) v}{l f\left(x_{1}, v_{1}\right) v_{1}}\right)+(m+\rho+\gamma) l_{1}\left(1-\frac{l y_{1}}{l_{1} y}\right)+(m+\rho+\gamma) l_{1}\left(1-\frac{v}{v_{1}}-\frac{y v_{1}}{y_{1} v}\right)+\rho\left(l-l_{1}\right) \\
& \cdot\left(1-\frac{f\left(x_{1}, v_{1}\right)}{f\left(x, v_{1}\right)}\right)-\frac{\rho\left(\alpha_{0}+\alpha_{2} v_{1}\right)\left[d\left(x-x_{1}\right)^{2}+(m+\gamma)\left(l-l_{1}\right)^{2}+(d+m+\gamma)\left(x-x_{1}\right)\left(l-l_{1}\right)\right]}{(d+m+\gamma)\left(\alpha_{0}+\alpha_{1} x_{1}+\alpha_{2} v_{1}+\alpha_{3} x_{1} v_{1}\right) x_{1}} \\
+ & \frac{a q h(m+\rho+\gamma)}{k g \gamma}\left(\frac{g v_{1}}{h}-1\right) w .
\end{aligned}
$$

Hence,

$$
\begin{aligned}
& D^{\alpha} L_{1}(t) \\
& \quad \leq-\frac{\left(\alpha_{0}+\alpha_{2} v_{1}\right)\left(x-x_{1}\right)^{2}}{x x_{1}\left(\alpha_{0}+\alpha_{1} x_{1}+\alpha_{2} v_{1}+\alpha_{3} x_{1} v_{1}\right)}\left(\left(d x_{1}-\rho l_{1}\right)+\rho l\right. \\
& \left.\quad+\frac{d \rho x}{d+m+\gamma}\right)
\end{aligned}
$$

$$
\begin{aligned}
& -\frac{\rho\left(\alpha_{0}+\alpha_{2} v_{1}\right)(m+\gamma)\left(l-l_{1}\right)^{2}}{(m+\rho+\gamma)\left(\alpha_{0}+\alpha_{1} x_{1}+\alpha_{2} v_{1}+\alpha_{3} x_{1} v_{1}\right) x_{1}}+(m+\rho \\
& +\gamma) l_{1}\left(5-\frac{f\left(x_{1}, v_{1}\right)}{f\left(x, v_{1}\right)}-\frac{l_{1} f(x, v) v}{l f\left(x_{1}, v_{1}\right) v_{1}}-\frac{l y_{1}}{l_{1} y}-\frac{y v_{1}}{y_{1} v}\right. \\
& \left.-\frac{f\left(x, v_{1}\right)}{f(x, v)}\right)-(m+\rho+\gamma) l_{1}
\end{aligned}
$$




$$
\begin{aligned}
& \cdot \frac{\left(\alpha_{0}+\alpha_{1} x\right)\left(\alpha_{2}+\alpha_{3} x\right)\left(v-v_{1}\right)^{2}}{v_{1}\left(\alpha_{0}+\alpha_{1} x+\alpha_{2} v+\alpha_{3} x v\right)\left(\alpha_{0}+\alpha_{1} x+\alpha_{2} v_{1}+\alpha_{3} x v_{1}\right)} \\
& +\frac{a q h(m+\rho+\gamma)}{k g \gamma}\left(R_{1}-1\right) w .
\end{aligned}
$$

Using the arithmetic-geometric inequality, we have

$$
5-\frac{f\left(x_{i}, v_{i}\right)}{f\left(x, v_{i}\right)}-\frac{l_{i} f(x, v) v}{l f\left(x_{i}, v_{i}\right) v_{i}}-\frac{l y_{i}}{l_{i} y}-\frac{y v_{i}}{y_{i} v}-\frac{f\left(x, v_{i}\right)}{f(x, v)}
$$

$$
\leq 0 \text {. }
$$

Since $R_{1} \leq 1$, we have $D^{\alpha} L_{1}(t) \leq 0$ if $d x_{1} \geq \rho l_{1}$. It is easy to see that this condition is equivalent to (44). Furthermore,
$D^{\alpha} L_{1}(t)=0$ if and only if $x=x_{1}, l=l_{1}, y=y_{1}, v=v_{1}$, and $\left(R_{1}-1\right) w=0$. We discuss two cases: If $R_{1}<1$, then $w=0$. If $R_{1}=1$, from (1), we get $D^{\alpha} v_{1}=0=k y_{1}-\mu v_{1}-q v_{1} w$, and then $w=0$. Hence, the largest invariant set of $\left\{(x, l, y, v, w) \in \mathbb{R}_{+}^{5}\right.$ : $\left.D^{\alpha} L_{1}(t)=0\right\}$ is the singleton $\left\{E_{1}\right\}$. By the LaSalle's invariance principle, $E_{1}$ is globally asymptotically stable.

At $E_{1}$, the characteristic equation of (40) is given as follows:

$$
\left(g v_{1}-h-\xi\right) g_{1}(\xi)=0
$$

where

$$
g_{1}(\xi)=\left|\begin{array}{cccc}
-d-\frac{\partial f}{\partial x}\left(x_{1}, v_{1}\right) v_{1}-\xi & \rho & 0 & -\frac{\partial f}{\partial v}\left(x_{1}, v_{1}\right) v_{1}-f\left(x_{1}, v_{1}\right) \\
\frac{\partial f}{\partial x}\left(x_{1}, v_{1}\right) v_{1} & -(m+\rho+\gamma)-\xi & 0 & \frac{\partial f}{\partial v}\left(x_{1}, v_{1}\right) v_{1}+f\left(x_{1}, v_{1}\right) \\
0 & \gamma & -a-\xi & 0 \\
0 & 0 & k & -\mu-\xi
\end{array}\right| .
$$

We can easily see that (50) has the root $\xi_{1}=g v_{1}-h$. Then, when $R_{1}>1$, we have $\xi_{1}>0$. In this case, $E_{1}$ is unstable.

Theorem 5. The infection equilibrium with humoral immunity $E_{2}$ is globally asymptotically stable if $R_{1}>1$ and

$$
\rho \beta h \leq d(m+\rho+\gamma)\left(\alpha_{0} g+\alpha_{2} h\right)+\rho \lambda\left(\alpha_{1} g+\alpha_{3} h\right) .
$$

Proof. Consider the following Lyapunov functional:

$$
\begin{aligned}
& L_{2}(t)=\frac{\alpha_{0}+\alpha_{2} v_{2}}{\alpha_{0}+\alpha_{1} x_{2}+\alpha_{2} v_{2}+\alpha_{3} x_{2} v_{2}} x_{2} \Phi\left(\frac{x}{x_{2}}\right) \\
& \quad+l_{2} \Phi\left(\frac{l}{l_{2}}\right)
\end{aligned}
$$

$$
\begin{aligned}
& +\frac{\rho\left(\alpha_{0}+\alpha_{2} v_{2}\right)}{2(d+m+\gamma)\left(\alpha_{0}+\alpha_{1} x_{2}+\alpha_{2} v_{2}+\alpha_{3} x_{2} v_{2}\right) x_{2}}(x \\
& \left.-x_{2}+l-l_{2}\right)^{2}+\frac{m+\rho+\gamma}{\gamma} y_{2} \Phi\left(\frac{y}{y_{2}}\right) \\
& +\frac{a(m+\rho+\gamma)}{k \gamma} v_{2} \Phi\left(\frac{v}{v_{2}}\right)+\frac{a q(m+\rho+\gamma)}{k g \gamma} \\
& \cdot w_{2} \Phi\left(\frac{w}{w_{2}}\right) .
\end{aligned}
$$

Computing the fractional derivative of $L_{2}(t)$ and using $\lambda=$ $d x_{2}+(m+\gamma) l_{2}, f\left(x_{2}, v_{2}\right) v_{2}=(m+\rho+\gamma) l_{2}, \gamma l_{2}=a y_{2}, k y_{2}=$ $\left(\mu+q w_{2}\right) v_{2}$, and $v_{2}=h / g$, we get

$$
\begin{aligned}
D^{\alpha} L_{2}(t) \leq & d\left(1-\frac{f\left(x_{2}, v_{2}\right)}{f\left(x, v_{2}\right)}\right)\left(x_{2}-x\right)+(m+\rho+\gamma) l_{2}\left(1-\frac{f\left(x_{2}, v_{2}\right)}{f\left(x, v_{2}\right)}+\frac{f(x, v) v}{f\left(x, v_{2}\right) v_{2}}\right) \\
& +(m+\rho+\gamma) l_{2}\left(1-\frac{l_{2} f(x, v) v}{l f\left(x_{2}, v_{2}\right) v_{2}}\right)+(m+\rho+\gamma) l_{2}\left(1-\frac{l_{2}}{l_{2} y}\right)+(m+\rho+\gamma) l_{2}\left(1-\frac{v}{v_{2}}-\frac{y v_{2}}{y_{2} v}\right) \\
& +\rho\left(l-l_{2}\right)\left(1-\frac{f\left(x_{2}, v_{2}\right)}{f\left(x, v_{2}\right)}\right) \\
& -\frac{\rho\left(\alpha_{0}+\alpha_{2} v_{2}\right)\left[d\left(x-x_{2}\right)^{2}+(m+\gamma)\left(l-l_{2}\right)^{2}+(d+m+\gamma)\left(x-x_{2}\right)\left(l-l_{2}\right)\right]}{(d+m+\gamma)\left(\alpha_{0}+\alpha_{1} x_{2}+\alpha_{2} v_{2}+\alpha_{3} x_{2} v_{2}\right) x_{2}}
\end{aligned}
$$




$$
\begin{aligned}
\leq & -\frac{\left(\alpha_{0}+\alpha_{2} v_{2}\right)\left(x-x_{2}\right)^{2}}{x x_{2}\left(\alpha_{0}+\alpha_{1} x_{2}+\alpha_{2} v_{2}+\alpha_{3} x_{2} v_{2}\right)}\left(\left(d x_{2}-\rho l_{2}\right)+\rho l+\frac{d \rho x}{d+m+\gamma}\right) \\
& -\frac{\rho\left(\alpha_{0}+\alpha_{2} v_{2}\right)(m+\gamma)\left(l-l_{2}\right)^{2}}{(m+\rho+\gamma)\left(\alpha_{0}+\alpha_{1} x_{2}+\alpha_{2} v_{2}+\alpha_{3} x_{2} v_{2}\right) x_{2}} \\
& +(m+\rho+\gamma) l_{2}\left(5-\frac{f\left(x_{2}, v_{2}\right)}{f\left(x, v_{2}\right)}-\frac{l_{2} f(x, v) v}{l f\left(x_{2}, v_{2}\right) v_{2}}-\frac{l y_{2}}{l_{2} y}-\frac{y v_{2}}{y_{2} v}-\frac{f\left(x, v_{2}\right)}{f(x, v)}\right) \\
& -(m+\rho+\gamma) l_{2} \frac{\left(\alpha_{0}+\alpha_{1} x\right)\left(\alpha_{2}+\alpha_{3} x\right)\left(v-v_{2}\right)^{2}}{v_{2}\left(\alpha_{0}+\alpha_{1} x+\alpha_{2} v+\alpha_{3} x v\right)\left(\alpha_{0}+\alpha_{1} x+\alpha_{2} v_{2}+\alpha_{3} x v_{2}\right)} .
\end{aligned}
$$

From (49), we have $D^{\alpha} L_{2}(t) \leq 0$ when $d x_{2} \geq \rho l_{2}$. This condition is equivalent to (52). In addition, $D^{\alpha} L_{2}(t)=0$ if $x=x_{2}, l=l_{2}, y=y_{2}$, and $v=v_{2}$. Further, $D^{\alpha} v_{2}=$ $0=k y_{2}-\mu v_{2}-q v_{2} w$; then $w=w_{2}$. Consequently, the largest invariant set of $\left\{(x, l, y, v, w) \in \mathbb{R}_{+}^{5}: D^{\alpha} L_{2}(t)=0\right\}$ is the singleton $\left\{E_{2}\right\}$. By the LaSalle's invariance principle, $E_{2}$ is globally asymptotically stable.

It is important to note that when $\rho$ is sufficiently small or $\gamma$ is sufficiently large, the two conditions (44) and (52) are satisfied. Then, we have the following corollary.

Corollary 6. Assume that $R_{0}>1$. When $\rho$ is sufficiently small or $\gamma$ is sufficiently large, then we have the following:

(i) The infection equilibrium without humoral immunity $E_{1}$ is globally asymptotically stable if $R_{1} \leq 1$.

(ii) The infection equilibrium with humoral immunity $E_{2}$ is globally asymptotically stable if $R_{1}>1$.

\section{Numerical Simulations}

In this section, we validate our theoretical results to HIV infection. Firstly, we take the parameter values as shown in Table 1.

By calculation, we have $R_{0}=0.4274 \leq 1$. Then system (1) has an infection-free equilibrium $E_{0}(719.4245,0,0,0,0)$. By Theorem 3, the solution of (1) converges to $E_{0}$ (see Figure 1). Consequently, the virus is cleared and the infection dies out.

Now, we choose $\beta=0.0012$ and we keep the other parameter values. Hence, we obtain $R_{0}=2.137, R_{1}=0.8334$, and

$$
\begin{aligned}
& 1+\frac{(m+\rho+\gamma)\left[\alpha_{0} a d \mu(m+\rho)+d k \lambda \gamma \alpha_{2}\right]+k \rho \gamma \alpha_{3} \lambda^{2}}{a \rho \mu(m+\rho+\gamma)\left(\alpha_{0} d+\lambda \alpha_{1}\right)} \\
& =2.5934 .
\end{aligned}
$$

Consequently, condition (44) is satisfied. Therefore, the infection equilibrium without humoral immunity $E_{1}(176.6853$, $168.7712,6.2508,1666.9,0)$ is globally asymptotically stable. Figure 2 demonstrates this result. In this case, the infection becomes chronic.
Next, we take $g=0.0004$ and do not change the other parameter values. In this case, we have $R_{1}=3.3338, \rho \beta h=$ 0.0000024 , and $d(m+\rho+\gamma)\left(\alpha_{0} g+\alpha_{2} h\right)+\rho \lambda\left(\alpha_{1} g+\alpha_{3} h\right)=$ 0.000006 . Hence, condition (52) is satisfied. Consequently, system (1) has an infection equilibrium with humoral immunity $E_{2}(423.4261,92.0442,3.4090,500,245.4473)$ which is globally asymptotically stable. Figure 3 illustrates this result. We can observe that the activation of the humoral immune response increases the healthy cells and decreases the productive infected cells and viral load to a lower levels but it is not able to eradicate the infection.

\section{Conclusion}

In the present paper, we have studied the dynamics of a viral infection model by taking into account the memory effect represented by the Caputo fractional derivative and the humoral immunity. We have proved that the solutions of the model are nonnegative and bounded which assure the well-posedness. We have shown that the proposed model has three infection equilibriums, namely, the infection-free equilibrium $E_{0}$, the infection equilibrium without humoral immunity $E_{1}$, and the infection equilibrium with humoral immunity $E_{2}$. By constructing suitable Lyapunov functionals, the global stability of these equilibria is fully determined by two threshold parameters $R_{0}$ and $R_{1}$. More precisely, when $R_{0} \leq 1, E_{0}$ is globally asymptotically stable, whereas if $R_{0}>1$, it becomes unstable and another equilibrium point appears, that is, $E_{1}$, which is globally asymptotically stable whenever $R_{1} \leq 1$ and condition (44) is satisfied. In the case that $R_{1}>1, E_{1}$ becomes unstable and there exists another equilibrium point $E_{2}$ which is globally asymptotically stable when condition (52) is satisfied. In addition, we remarked that when $\rho$ is sufficiently small or $\gamma$ is sufficiently large, conditions (44) and (52) are verified, and then the global stability of $E_{1}$ and $E_{2}$ is characterized only by $R_{0}$ and $R_{1}$.

From our theoretical and numerical results, we deduce that the order of the fractional derivative $\alpha$ has no effect on the dynamics of the model. However, when the value of $\alpha$ decreases (long memory), the solutions of our model converge rapidly to the steady states (see Figures $1-3$ ). This behavior can be explained by the memory term $1 / \Gamma(1-\alpha)(t-$ $u)^{\alpha}$ included in the fractional derivative which represents 
TABLE 1: Parameter values of system (1).

\begin{tabular}{lccccc}
\hline parameters & values & parameters & values & parameters & values \\
\hline$\lambda$ & 10 & $a$ & 0.27 & 0.2 \\
$d$ & 0.0139 & $\gamma$ & 0.01 & $g$ & 0.0001 \\
$\beta$ & 0.00024 & $k$ & 800 & $\alpha_{0}$ & 1 \\
$\rho$ & 0.01 & $\mu$ & 3 & $\alpha_{1}$ & 0.1 \\
$m$ & 0.0347 & $q$ & 0.01 & $\alpha_{2}$ & 0.01 \\
\hline
\end{tabular}
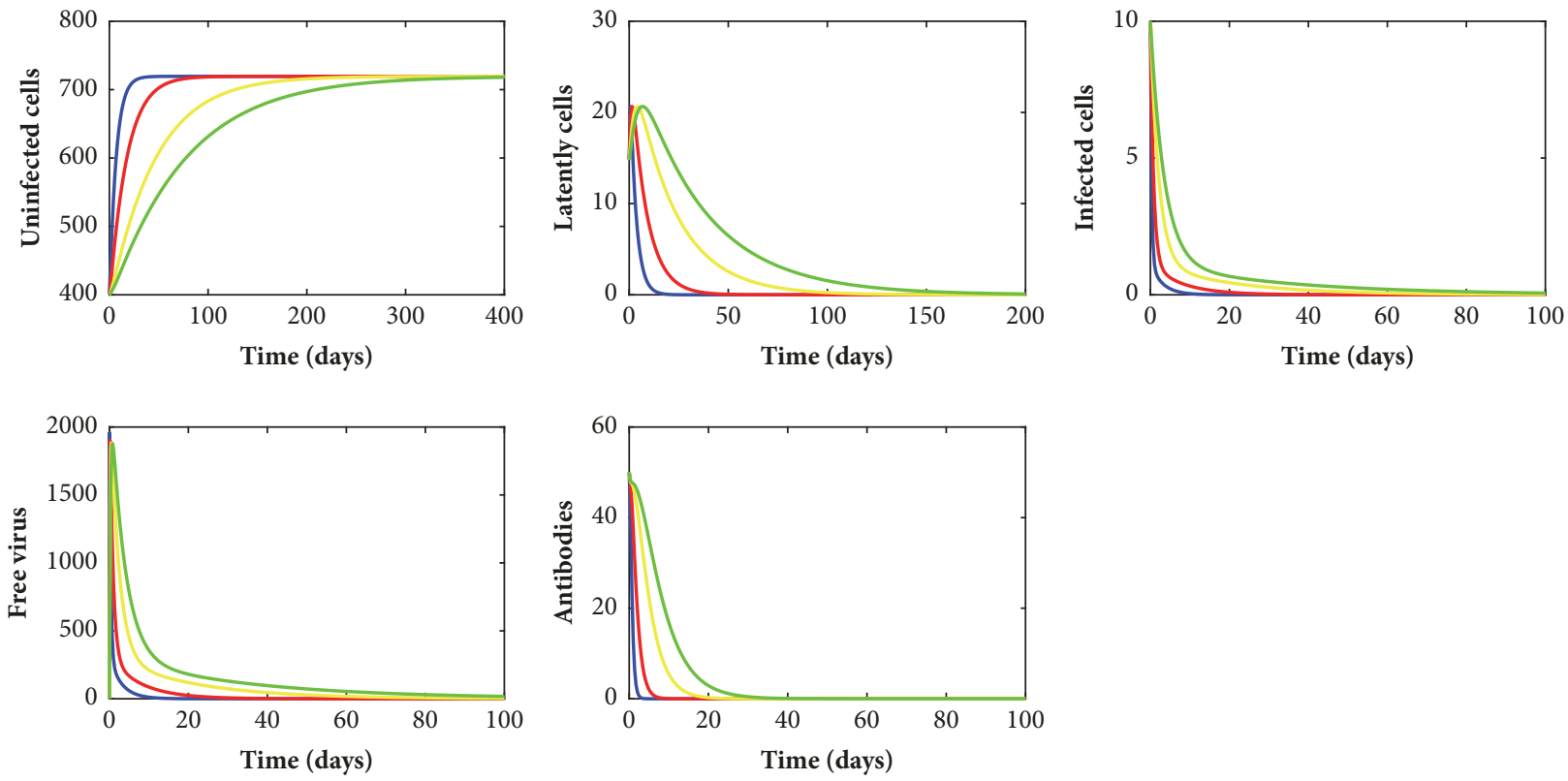

$\alpha=0.5$

$\alpha=0.7$

$\alpha=0.9$

$\alpha=1$

FIGURE 1: Stability of the infection-free equilibrium $E_{0}$.
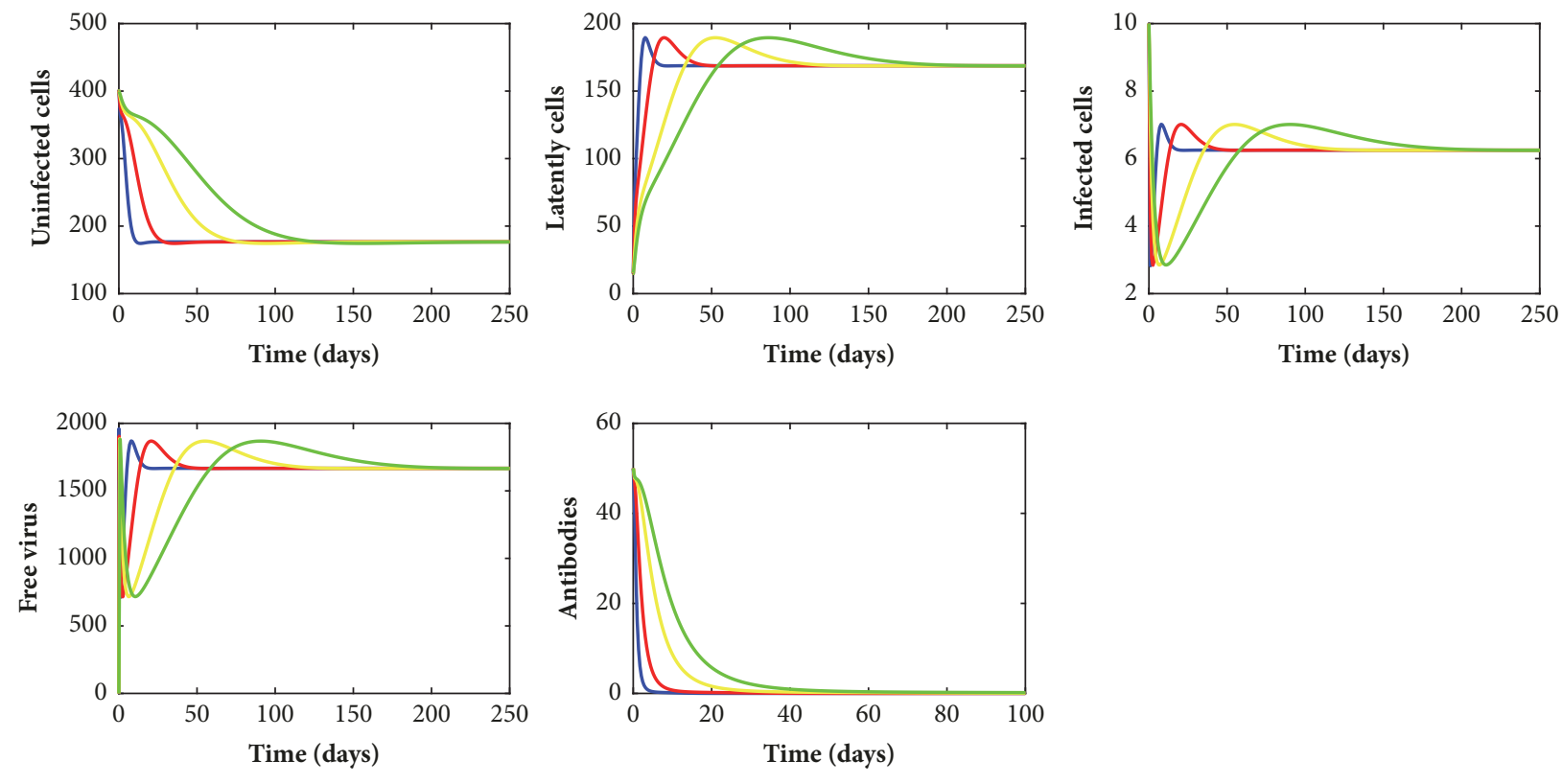

FIgURE 2: Stability of the infection equilibrium without humoral immunity $E_{1}$. 

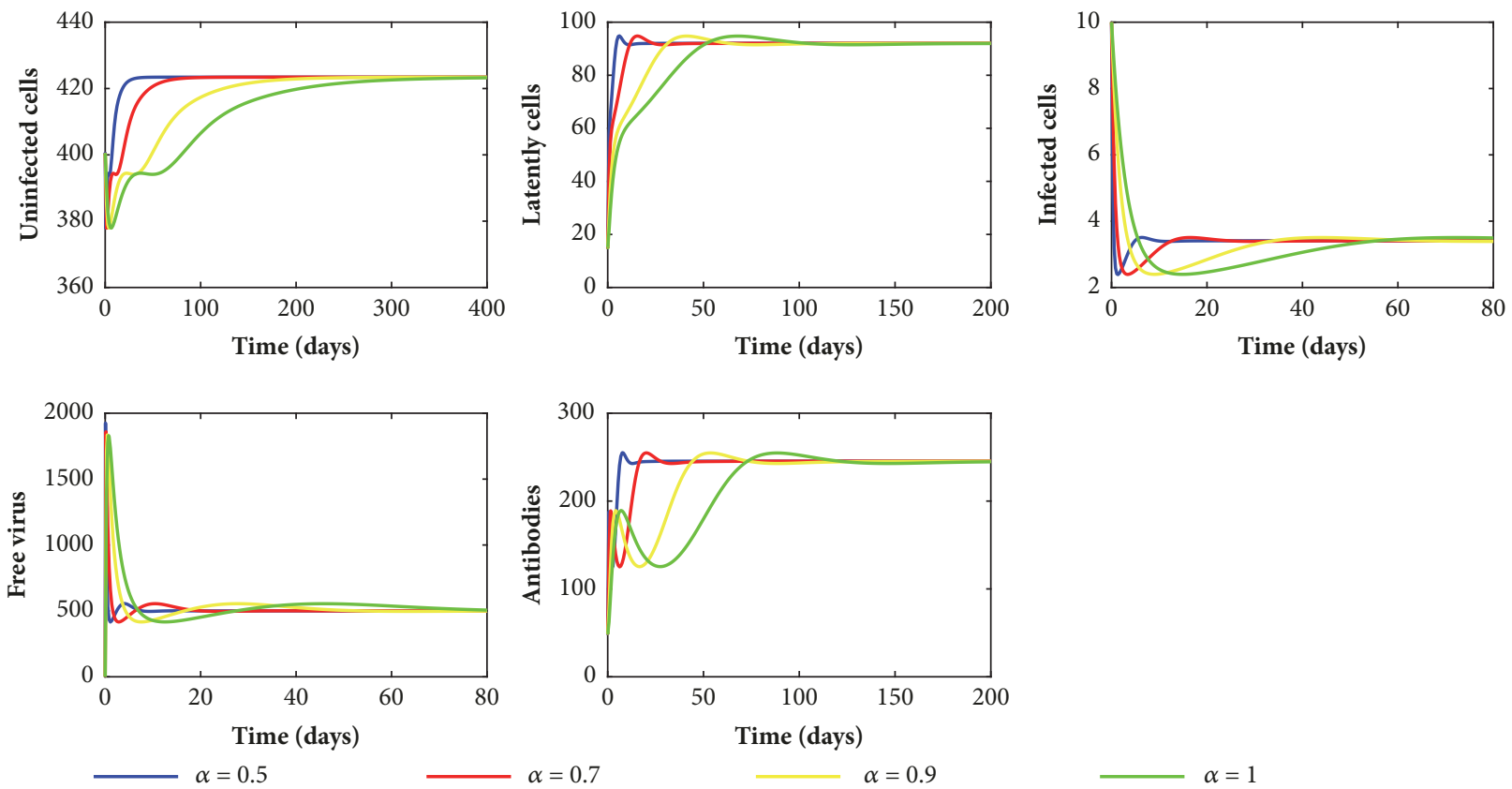

FIGURE 3: Stability of the infection equilibrium with humoral immunity $E_{2}$.

the time needed for the interaction between cells and viral particles and the time needed for the activation of humoral immune response. In fact, the knowledge about the infection and the activation of the humoral immune response in an early stage can help us to control the infection.

\section{Data Availability}

The data used to support the findings of this study are available from the corresponding author upon request.

\section{Conflicts of Interest}

The authors declare that they have no conflicts of interest.

\section{References}

[1] K. Hattaf, N. Yousfi, and A. Tridane, "Global stability analysis of a generalized virus dynamics model with the immune response," Canadian Applied Mathematics Quarterly, vol. 20, no. 4, pp. 499-518, 2012.

[2] M. Maziane, K. Hattaf, and N. Yousfi, "Global stability for a class of HIV infection models with cure of infected cells in eclipse stage and CTL immune response," International Journal of Dynamics and Control, 2016.

[3] X. Wang, Y. Tao, and X. Song, "Global stability of a virus dynamics model with Beddington-DeAngelis incidence rate and CTL immune response," Nonlinear Dynamics, vol. 66, no. 4, pp. 825-830, 2011.

[4] C. Lv, L. Huang, and Z. Yuan, "Global stability for an HIV-1 infection model with Beddington-DeAngelis incidence rate and CTL immune response," Communications in Nonlinear Science and Numerical Simulation, vol. 19, no. 1, pp. 121-127, 2014.
[5] M. A. Nowak and C. R. M. Bangham, "Population dynamics of immune responses to persistent viruses," Science, vol. 272, no. 5258, pp. 74-79, 1996.

[6] A. Murase, T. Sasaki, and T. Kajiwara, "Stability analysis of pathogen-immune interaction dynamics," Journal of Mathematical Biology, vol. 51, no. 3, pp. 247-267, 2005.

[7] M. A. Obaid and A. M. Elaiw, "Stability of Virus Infection Models with Antibodies and Chronically Infected Cells," Abstract and Applied Analysis, vol. 2014, Article ID 650371, 12 pages, 2014.

[8] M. A. Obaid, "Dynamical behaviors of a nonlinear virus infection model with latently infected cells and immune response," Journal of Computational Analysis and Applications, vol. 21, no. 1, pp. 182-193, 2016.

[9] H. F. Huo, Y. L. Tang, and L. X. Feng, "A Virus Dynamics Model with Saturation Infection and Humoral Immunity," Int. Journal of Math. Analysis, vol. 6, no. 40, 2012.

[10] A. M. Elaiw, "Global stability analysis of humoral immunity virus dynamics model including latently infected cells," Journal of Biological Dynamics, vol. 9, no. 1, pp. 215-228, 2015.

[11] A. M. Elaiw and N. H. AlShamrani, "Global properties of nonlinear humoral immunity viral infection models," International Journal of Biomathematics, vol. 8, no. 5, 1550058, 53 pages, 2015.

[12] J. X. Velasco-Herna'ndez, J. A. Garci'a, and D. E. Kirschner, "Remarks on modeling host-viral dynamics and treatment, Mathematical Approaches for Emerging and Reemerging Infectious Diseases," An Introduction to Models, Methods, and Theory, vol. 125, pp. 287-308, 2002.

[13] A. S. Perelson, "Modelling viral and immune system dynamics," Nature Reviews Immunology, vol. 2, no. 1, pp. 28-36, 2002.

[14] R. L. Magin, "Fractional calculus models of complex dynamics in biological tissues," Computers \& Mathematics with Applications, vol. 59, no. 5, pp. 1586-1593, 2010.

[15] A. A. Stanislavsky, "Memory effects and macroscopic manifestation of randomness," Physical Review E: Statistical Physics, 
Plasmas, Fluids, and Related Interdisciplinary Topics, vol. 61, no. 5, pp. 4752-4759, 2000.

[16] M. Saeedian, M. Khalighi, N. Azimi-Tafreshi, G. R. Jafari, and M. Ausloos, "Memory effects on epidemic evolution: The susceptible-infected-recovered epidemic model," Physical Review E: Statistical, Nonlinear, and Soft Matter Physics, vol. 95, no. 2, Article ID 022409, 2017.

[17] Y. A. Rossikhin and M. V. Shitikova, "Applications of fractional calculus to dynamic problems of linear and nonlinear hereditary mechanics of solids," Applied Mechanics Reviews, vol. 50, no. 1, pp. 15-67, 1997.

[18] R. J. Marks and M. W. Hall, "Differintegral Interpolation from a Bandlimited Signal's Samples," IEEE Transactions on Signal Processing, vol. 29, no. 4, pp. 872-877, 1981.

[19] G. L. Jia and Y. X. Ming, "Study on the viscoelasticity of cancellous bone based on higher-order fractional models," in Proceedings of the 2nd International Conference on Bioinformatics and Biomedical Engineering (ICBBE '08), pp. 1733-1736, Shanghai, China, May 2008.

[20] R. Magin, "Fractional calculus in bioengineering," Cretical reviews in biomedical engineering, vol. 32, pp. 13-77, 2004.

[21] E. Scalas, R. Gorenflo, and F. Mainardi, "Fractional calculus and continuous-time finance," Physica A: Statistical Mechanics and its Applications, vol. 284, no. 1-4, pp. 376-384, 2000.

[22] R. Capponetto, G. Dongola, L. Fortuna, and I. Petras, "Fractional order systems: Modelling and control applications," World Scientific Series in Nonlinear Science, Series A, vol. 72, 2010.

[23] V. D. Djordjević, J. Jarić, B. Fabry, J. J. Fredberg, and D. Stamenović, "Fractional derivatives embody essential features of cell rheological behavior," Annals of Biomedical Engineering, vol. 31, no. 6, pp. 692-699, 2003.

[24] K. S. Cole, "Electric conductance of biological systems," Cold Spring Harbor Symposium on Quantitative Biology, vol. 1, pp. 107-116, 1933.

[25] F. A. Rihan, M. Sheek-Hussein, A. Tridane, and R. Yafia, "Dynamics of hepatitis C virus infection: mathematical modeling and parameter estimation," Mathematical Modelling of Natural Phenomena, vol. 12, no. 5, pp. 33-47, 2017.

[26] N. Khodabakhshi, S. M. Vaezpour, and D. Baleanu, "On Dynamics Of a Fractional-order Model Of HCV Infection," Journal of Mathematical Analysis, vol. 8, no. 1, pp. 16-27, 2017.

[27] X. Zhou and Q. Sun, "Stability analysis of a fractional-order HBV infection model," International Journal of Advances in Applied Mathematics and Mechanics, vol. 2, no. 2, pp. 1-6, 2014.

[28] S. S. M. and Y. A. M., "On a fractional-order model for HBV infection with cure of infected cells," Journal of the Egyptian Mathematical Society, vol. 25, no. 4, pp. 445-451, 2017.

[29] Y. Ding and H. Ye, "A fractional-order differential equation model of HIV infection of CD4+ T-cells," Mathematical and Computer Modelling, vol. 50, no. 3-4, pp. 386-392, 2009.

[30] C. M. Pinto and A. R. Carvalho, "The role of synaptic transmission in a HIV model with memory," Applied Mathematics and Computation, vol. 292, pp. 76-95, 2017.

[31] A. Boukhouima, K. Hattaf, and N. Yousfi, "Dynamics of a Fractional Order HIV Infection Model with Specific Functional Response and Cure Rate," International Journal of Differential Equations, vol. 2017, Article ID 8372140, 8 pages, 2017.

[32] J. A. Deans and S. Cohen, "Immunology of malaria," Annual Review of Microbiology, vol. 37, pp. 25-49, 1983.
[33] I. Podlubny, Fractional Differential Equations: An Introduction to Fractional Derivatives, Fractional Differential Equations, to Methods of their Solution and Some of their Applications, vol. 198, Academic press, 1998.

[34] K. Hattaf and N. Yousfi, "A class of delayed viral infection models with general incidence rate and adaptive immune response," International Journal of Dynamics and Control, vol. 4, no. 3, pp. 254-265, 2016.

[35] D. Riad, K. Hattaf, and N. Yousfi, "Dynamics of Capital-labour Model with Hattaf-Yousfi Functional Response," British Journal of Mathematics \& Computer Science, vol. 18, no. 5, pp. 1-7, 2016.

[36] M. Mahrouf, K. Hattaf, and N. Yousfi, "Dynamics of a stochastic viral infection model with immune response," Mathematical Modelling of Natural Phenomena, vol. 12, no. 5, pp. 15-32, 2017.

[37] K. Hattaf, N. Yousfi, and A. Tridane, "Stability analysis of a virus dynamics model with general incidence rate and two delays," Applied Mathematics and Computation, vol. 221, pp. 514-521, 2013.

[38] P. Crowley and E. Martin, "Functional responses and interference within and between year classes of a dragonfly population," Journal of the North American Benthological Society, vol. 8, pp. 211-221, 1989.

[39] S. Xu, "Global stability of the virus dynamics model with Crowley-Martin functional response," Electronic Journal of Qualitative Theory of Differential Equations, No. 9, 10 pages, 2012.

[40] J. R. Beddington, "Mutual interference between parasites or predat ors and its effect on searching efficiency," Journal of Animal Ecology, vol. 44, pp. 331-340, 1975.

[41] D. L. DeAngelis, R. A. Goldstein, and R. V. ONeill, "A model for trophic interaction," Ecology, pp. 881-892, 1975.

[42] L. Rong, M. A. Gilchrist, Z. Feng, and A. S. Perelson, "Modeling within-host HIV-1 dynamics and the evolution of drug resistance: trade-offs between viral enzyme function and drug susceptibility," Journal of Theoretical Biology, vol. 247, no. 4, pp. 804-818, 2007.

[43] Z. Hu, W. Pang, F. Liao, and W. Ma, "Analysis of a cd4+ t cell viral infection model with a class of saturated infection rate," Discrete and Continuous Dynamical Systems - Series B, vol. 19, no. 3, pp. 735-745, 2014.

[44] M. Maziane, E. M. Lotfi, K. Hattaf, and N. Yousfi, "Dynamics of a Class of HIV Infection Models with Cure of Infected Cells in Eclipse Stage," Acta Biotheoretica, vol. 63, no. 4, pp. 363-380, 2015.

[45] J. K. Hale and S. M. Verduyn Lunel, Introduction to FunctionalDifferential Equations, Springer, Berlin, Germany, 1993.

[46] J. K. Hale, "Sufficient conditions for stability and instability of autonomous functional-differential equations," Journal of Differential Equations, vol. 1, pp. 452-482, 1965.

[47] T. A. Burton, Stability and Periodic sSolutions of Ordinary and Functional Differential Equations, Academic Press, Orlando, Fla, USA, 1985.

[48] B. S. Ogundare, "Stability and boundedness properties of solutions to certain fifth order nonlinear differential equations," Matematicki Vesnik, vol. 61, no. 4, pp. 257-268, 2009.

[49] C. Tunç, "A study of the stability and boundedness of the solutions of nonlinear differential equations of the fifth order," Indian Journal of Pure and Applied Mathematics, vol. 33, no. 4, pp. 519-529, 2002.

[50] C. Tunç, "New results on the stability and boundedness of nonlinear differential equations of fifth order with multiple 
deviating arguments," Bulletin of the Malaysian Mathematical Sciences Society, vol. 36, no. 3, pp. 671-682, 2013.

[51] J. Huo, H. Zhao, and L. Zhu, "The effect of vaccines on backward bifurcation in a fractional order HIV model," Nonlinear Analysis: Real World Applications, vol. 26, pp. 289-305, 2015.

[52] C. Vargas-De-Leon, "Volterra-type Lyapunov functions for fractional-order epidemic systems," Communications in Nonlinear Science and Numerical Simulation, vol. 24, no. 1-3, pp. 75-85, 2015.

[53] D. Matignon, "Stability results for fractional differential equations with applications to control processing," in Computational Eng. in Sys. Appl, vol. 2, p. 963, Lille, France, 1996.

[54] E. Ahmed, A. M. A. El-Sayed, and H. A. A. El-Saka, "On some Routh-Hurwitz conditions for fractional order differential equations and their applications in Lorenz, Rössler, Chua and Chen systems," Physics Letters A, vol. 358, no. 1, pp. 1-4, 2006.

[55] E. Ahmed, A. M. A. El-Sayed, and H. A. A. El-Saka, "Equilibrium points, stability and numerical solutions of fractionalorder predator-prey and rabies models," Journal of Mathematical Analysis and Applications, vol. 325, no. 1, pp. 542-553, 2007. 


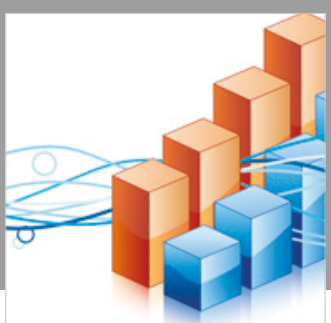

Advances in

Operations Research

\section{-n-m}
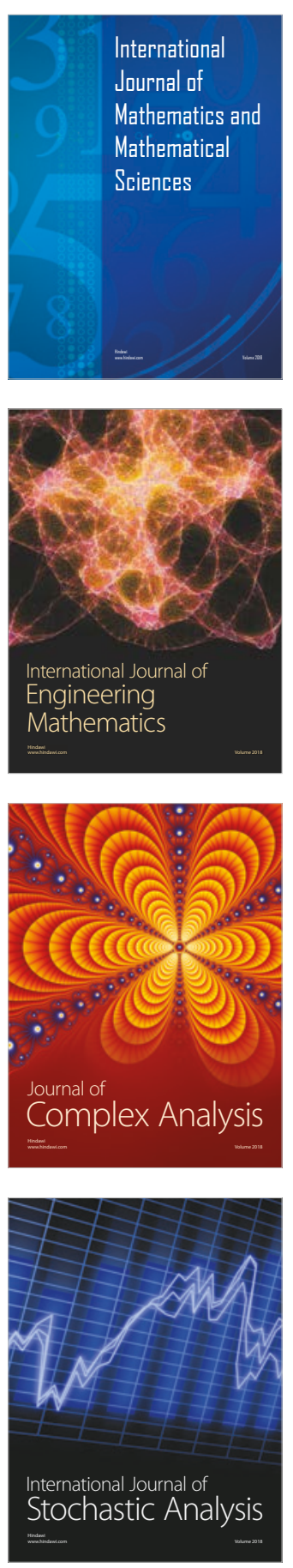
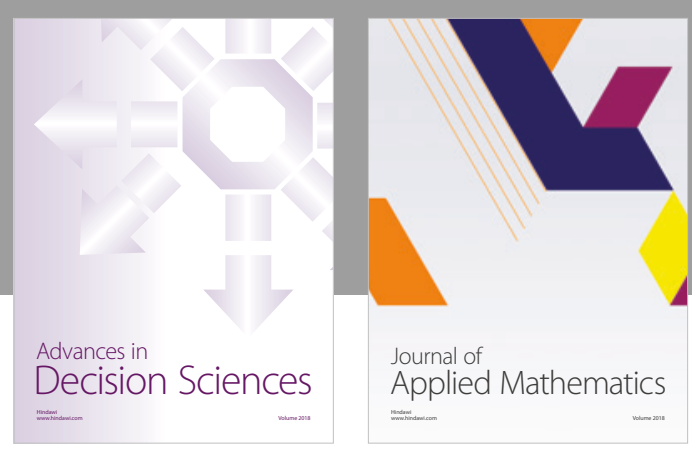

Journal of

Applied Mathematics
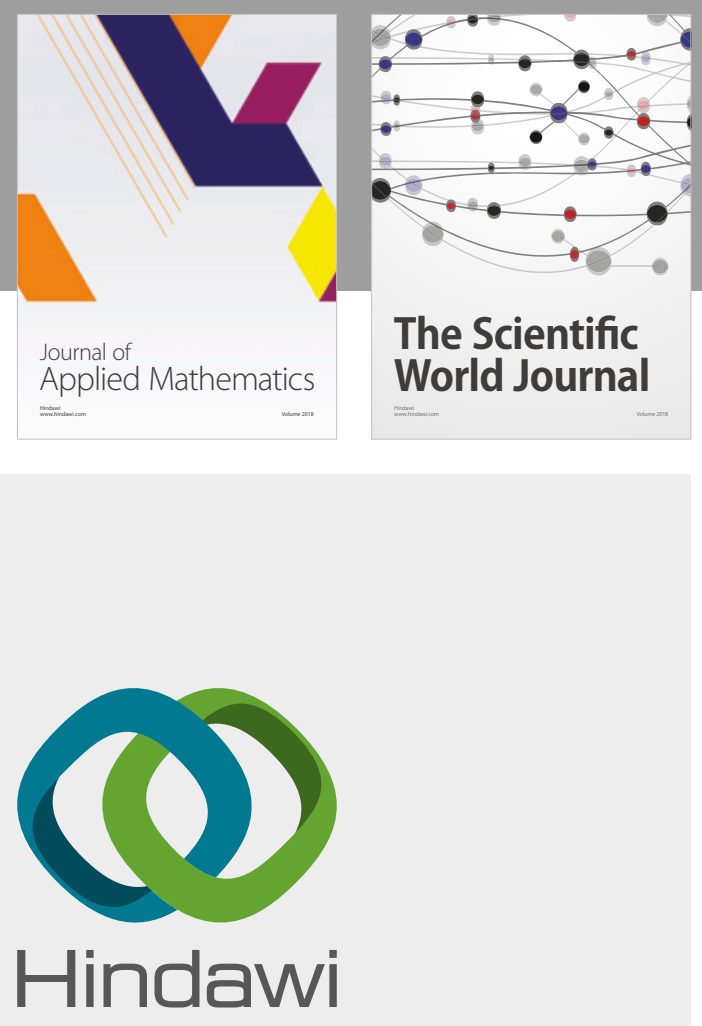

Submit your manuscripts at

www.hindawi.com

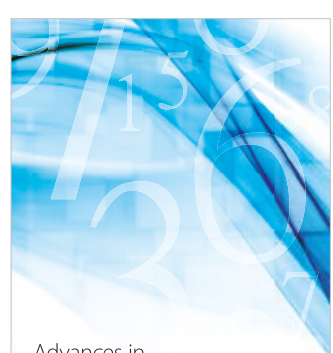

Advances in
Numerical Analysis
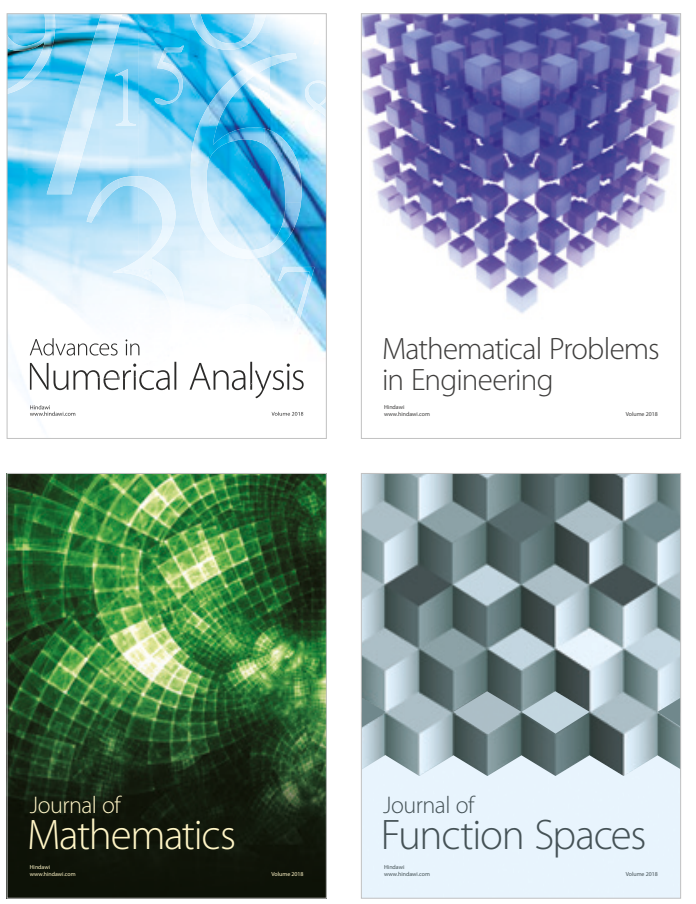

Mathematical Problems in Engineering

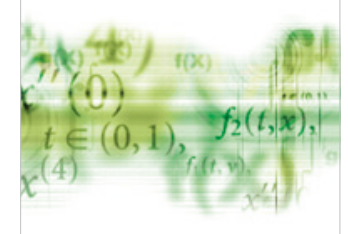

International Journal of

Differential Equations

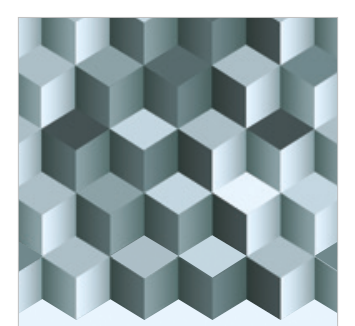

Journal of

Function Spaces

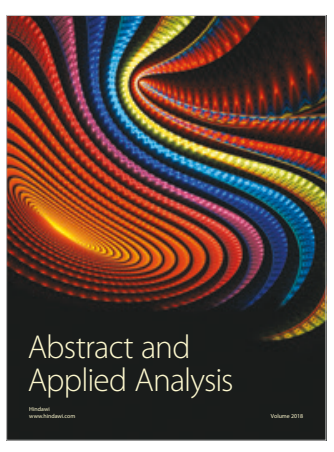

The Scientific

World Journal

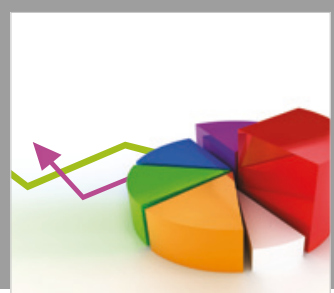

Journal of

Probability and Statistics
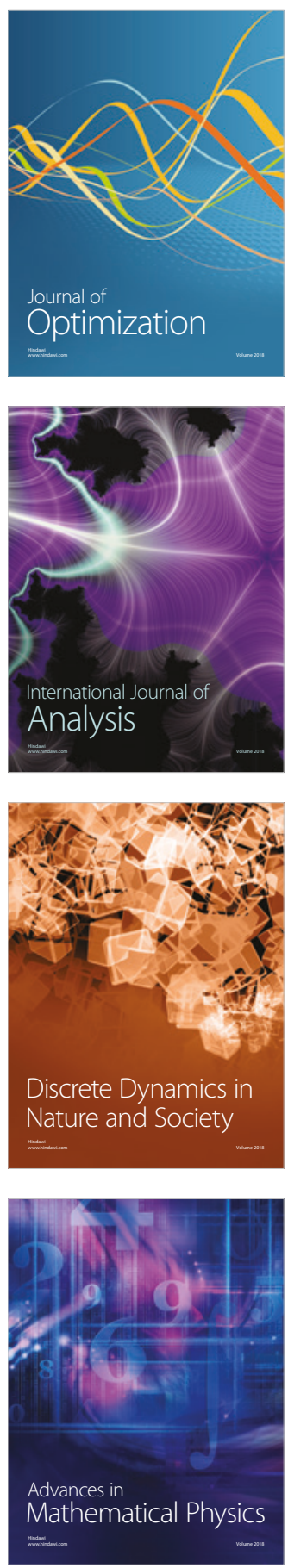